\title{
PREDICTIVE CONTROL FOR THE ALSTOM
}

\section{GASIFIER PROBLEM}

\author{
R K Al Seyab ${ }^{\ddagger} \quad$ Y Cao $^{\ddagger *} \quad$ S H Yang $^{\dagger}$ \\ ${ }^{\ddagger}$ Cranfield University, UK \\ 'Loughborough University, UK
}

Keywords: Nonlinear Systems, Optimal Control, Predictive Control, Process Control, ALSTOM Gasifier

\begin{abstract}
Model Predictive Control (MPC) has become the first choice of control strategy in many cases especially in the process industry because it is intuitive and can explicitly handle MIMO systems with input and output constraints. In this paper, a simple MPC algorithm based on the state space formulation is implemented to control the ALSTOM gasifier. Among three operating conditions of the plant, $0 \%$ load condition is identified as the worst case. A linearized state space model at $0 \%$ load condition of the nonlinear plant is adopted as the internal model for performance prediction. Due to this choice, the control system comfortably achieves performance requirements at the most difficult load condition. Meanwhile, the case study shows that the model is also adequate to pass all tests under other load conditions specified in the benchmark problem. The MPC algorithm uses standard formulation and off-the-shelf software with a few tunable parameters. Thus, it is easy to implement and to tune to achieve satisfactory performance.
\end{abstract}

\footnotetext{
*To whom correspondence should be addressed (y.cao@cranfield.ac.uk).
} 


\section{Introduction}

The ALSTOM gasifier, a complicated nonlinear process, was issued as a benchmark control problem by the ALSTOM Power Technology Centre [1]. The control problem involves several challenging issues, such as high order, high nonlinearity and strong interactions among process variables. Furthermore, the process has very stringent constraints on the process variables because of safety and environmental issues and the physical nature of these variables themselves.

The benchmark challenge was issued in two stages. The first round challenge, which was issued in 1997, included three linear models representing three operating conditions of the gasifier at $0 \%, 50 \%$, and $100 \%$ load respectively. The challenge requires the gasifier to be controlled at these load conditions to satisfy certain input and output constraints in the presence of step and sinusoidal sink pressure disturbances. A detailed description of the ALSTOM gasifier with design specifications for this round challenge are available in [1]. Initial attempts to control the gasifier were presented at a meeting [2] held at Coventry University on 24th July 1998. An overview of various control strategies and their comparison was given in [2]. One of the issues remaining unsolved in the first round challenge was that all controllers discussed at the meeting violated the process constraints by various degrees. The only model predictive control (MPC) approach presented at the meeting [3] involved the use of an additional inner loop to stabilize the process. The inner loop controller was supervised by an outer loop to handle the process constraints. A linear model at $100 \%$ load condition was used as the internal model for the MPC controller. In a more recent work [4], a controller was designed using the $H_{2}$ methodology at $100 \%$ load condition. When comparing with other control strategies it was reported that "the controller is acceptable due to the small percentage of constraints violations".

The second round challenge was issued in 2002 [5]. The original challenge 
was extended by providing participants with a nonlinear simulation model of the gasifier in MATLAB/SIMULINK. In this round, in addition to the original disturbance tests and the unsolved constraint violation issue, two extra tests were added: load change test and coal quality disturbance test [5].

In this work, a simple linear MPC approach is chosen to control the gasifier because MPC is known for its capability to handle multivariable interaction and process constraints in the most natural way. In the predictive controller, a quadratic programming $(\mathrm{QP})$ problem is solved online to decide the optimum control moves to steer the output to follow a specified trajectory that keeps the process in the stable operating regions all the time. The novelty of the work is to identify the load condition at $0 \%$ as the most difficult case of all three operating conditions of the gasifier to achieve performance specifications. Then, a linear state space model around $0 \%$ load point is used as the internal model for performance prediction. The model works fairly well at other load levels. The controller is implemented by using quadprog function of MATLAB to solve the QP optimization problem. This allows different constraints to be easily handled by the controller. Good results are obtained satisfying all the tests requirement given in [5] without any constraint violation. Comparing to previous attempts proposed in [3, 4], the success of this work should contribute to the right decision of selecting a linear nominal (internal) model for nonlinear system control. Some intuitive thinking behind this decision will be explained in the paper. The full specification of the plant together with the control objectives and performance tests, have been described in the introduction paper of the present issue [5], hence is omitted in the paper. The rest of this paper is organized as follows. Section 2 gives formulations of the predictive controller to be used in the work. Details of control design are explained in section 3. Section 4 presents simulation results obtained with the nonlinear simulation model controlled by the MPC controller. In section 5, some conclusions are drawn from this work. 


\section{Predictive control formulation}

Assume that the plant considered has manipulable input, $\tilde{\boldsymbol{u}} \in \mathbb{R}^{n_{u}}$ and measured output, $\tilde{\boldsymbol{y}} \in \mathbb{R}^{n_{y}}$, which have steady-state values, $\tilde{\boldsymbol{u}}_{0}$ and $\tilde{\boldsymbol{y}}_{0}$ at the nominal operating point respectively. Around the operating point, the dynamic behaviour of the plant can be approximated by the following linear discrete-time state-space equations:

$$
\begin{aligned}
\boldsymbol{x}(k+1) & =\boldsymbol{A} \boldsymbol{x}(k)+\boldsymbol{B} \boldsymbol{u}(k) \\
\boldsymbol{y}(k) & =\boldsymbol{C} \boldsymbol{x}(k)+\boldsymbol{d}(k)
\end{aligned}
$$

where $k$ stands for $k$ th sampling time, $\boldsymbol{u}(k)=\tilde{\boldsymbol{u}}(k)-\tilde{\boldsymbol{u}}_{0}$ and $\boldsymbol{y}(k)=\tilde{\boldsymbol{y}}(k)-\tilde{\boldsymbol{y}}_{0}$ are deviation variables, $\boldsymbol{d}(k)$ the virtual disturbance estimated at output and $\boldsymbol{x}(k)$ the internal state of the model. The model and plant are assumed to be coincident at a nominal operating point at $t=0$. Hence, $\boldsymbol{x}(0)=0, \boldsymbol{u}(0)=0, \boldsymbol{y}(0)=0$ and $\boldsymbol{d}(0)=0$.

At $k$ th sampling time, with the currently measured output, $\boldsymbol{y}_{m}(k)=\tilde{\boldsymbol{y}}(k)-$ $\tilde{\boldsymbol{y}}_{0}$ and the current state of the internal model, $\boldsymbol{x}(k)$, the future output within the prediction horizon, $P$ can be estimated from the future input (to be determined within the moving horizon, $M), \boldsymbol{u}(k)$ as follows: assuming

$$
\boldsymbol{d}(k+i)=\boldsymbol{d}_{k}=\boldsymbol{y}_{m}(k)-\boldsymbol{C x}(k), \text { for } i=1, \ldots, P
$$

then

$$
\boldsymbol{Y}=\boldsymbol{\Phi} \boldsymbol{U}+\boldsymbol{\Psi} \boldsymbol{x}(k)+\boldsymbol{L} \boldsymbol{d}_{k}
$$


where

$$
\begin{aligned}
\boldsymbol{Y} & =\left[\begin{array}{lll}
\boldsymbol{y}^{T}(k+1) & \cdots & \boldsymbol{y}^{T}(k+P)
\end{array}\right]^{T} \\
\boldsymbol{U} & =\left[\begin{array}{llll}
\boldsymbol{u}^{T}(k) & \cdots & \boldsymbol{u}^{T}(k+M-1)
\end{array}\right]^{T} \\
\boldsymbol{\Phi} & =\left[\begin{array}{cccc}
\boldsymbol{C} \boldsymbol{B} & \mathbf{0} & \cdots & \mathbf{0} \\
\boldsymbol{C A} \boldsymbol{B} & \boldsymbol{C} \boldsymbol{B} & \cdots & \mathbf{0} \\
\vdots & \vdots & \cdots & \vdots \\
\boldsymbol{C} \boldsymbol{A}^{P-1} \boldsymbol{B} & \boldsymbol{C A}^{P-2} \boldsymbol{B} & \cdots & \sum_{i=M}^{P} \boldsymbol{C} \boldsymbol{A}^{P-i} \boldsymbol{B}
\end{array}\right] \\
\boldsymbol{\Psi} & =\left[\begin{array}{c}
\boldsymbol{C} \boldsymbol{A} \\
\vdots \\
\boldsymbol{C} \boldsymbol{A}^{P}
\end{array}\right] \\
\boldsymbol{L} & =\left[\begin{array}{lll}
\boldsymbol{I} & \cdots & \boldsymbol{I}
\end{array}\right]^{T}
\end{aligned}
$$

Future input, $\boldsymbol{U}$ is determined to follow the output reference, $\boldsymbol{y}_{r}(k)$, and the input reference $\boldsymbol{u}_{r}(k)=\boldsymbol{H}_{0}\left(\boldsymbol{y}_{r}(k)-\boldsymbol{d}_{k}\right)$, where $\boldsymbol{H}_{0}=\boldsymbol{C}(\boldsymbol{I}-\boldsymbol{A})^{-1} \boldsymbol{B}$. Define input and output reference vectors as

$$
\begin{aligned}
\boldsymbol{Y}_{r} & =\left[\begin{array}{lll}
\boldsymbol{y}_{r}^{T}(k+1) & \cdots & \boldsymbol{y}_{r}^{T}(k+P)
\end{array}\right]^{T} \\
\boldsymbol{U}_{r} & =\left[\begin{array}{lll}
\boldsymbol{u}_{r}^{T}(k) & \cdots & \boldsymbol{u}_{r}^{T}(k+M-1)
\end{array}\right]^{T}
\end{aligned}
$$

Then, $\boldsymbol{U}_{r}=\boldsymbol{H}\left(\boldsymbol{Y}_{r}-\boldsymbol{L} \boldsymbol{d}_{k}\right)$, where

$$
\boldsymbol{H}=\left[\begin{array}{ccccccc}
\boldsymbol{H}_{0} & \mathbf{0} & \cdots & \mathbf{0} & \mathbf{0} & \cdots & \mathbf{0} \\
\mathbf{0} & \boldsymbol{H}_{0} & \cdots & \mathbf{0} & \mathbf{0} & \cdots & \mathbf{0} \\
\vdots & \vdots & \cdots & \vdots & \vdots & \cdots & \vdots \\
\mathbf{0} & \mathbf{0} & \cdots & \boldsymbol{H}_{0} & \mathbf{0} & \cdots & \mathbf{0}
\end{array}\right]
$$


The optimization problem is to minimize the performance cost:

$$
\begin{aligned}
J= & 0.5\left(\boldsymbol{Y}-\boldsymbol{Y}_{r}\right)^{T} \boldsymbol{Q}\left(\boldsymbol{Y}-\boldsymbol{Y}_{r}\right)+0.5\left(\boldsymbol{U}-\boldsymbol{U}_{r}\right)^{T} \boldsymbol{R}\left(\boldsymbol{U}-\boldsymbol{U}_{r}\right) \\
\text { s.t. } \quad & \underline{\boldsymbol{u}} \leq \boldsymbol{u} \leq \overline{\boldsymbol{u}} \\
& |\boldsymbol{u}(k+1)-\boldsymbol{u}(k)| \leq \boldsymbol{\delta}_{u}
\end{aligned}
$$

where, output and input weighting matrices, $\boldsymbol{Q}$ and $\boldsymbol{R}$ are positive definite and $\underline{\boldsymbol{u}}$, $\overline{\boldsymbol{u}}$ and $\boldsymbol{\delta}_{u}$ are the lower, upper and maximum rate bounds of the input respectively.

Using the predictive equation (3), the optimization problem is equivalent to a standard quadratic programming $(\mathrm{QP})$ problem:

$$
\begin{array}{ll}
J= & 0.5 \boldsymbol{U}^{T} \boldsymbol{S} \boldsymbol{U}+\boldsymbol{U}^{T}\left(\boldsymbol{X}_{1} \boldsymbol{x}(k)-\boldsymbol{X}_{2}\left(\boldsymbol{Y}_{r}-\boldsymbol{L} \boldsymbol{d}_{k}\right)\right) \\
\text { s.t. } & \boldsymbol{U} \leq \overline{\boldsymbol{U}} \\
& -\boldsymbol{U} \leq-\underline{\boldsymbol{U}} \\
& \boldsymbol{E} \boldsymbol{U} \leq \boldsymbol{\Delta}_{u}+\boldsymbol{F} \boldsymbol{u}(k-1) \\
& -\boldsymbol{E} \boldsymbol{U} \leq \boldsymbol{\Delta}_{u}-\boldsymbol{F} \boldsymbol{u}(k-1)
\end{array}
$$

where, $\boldsymbol{u}(k-1)$ is the previous input, and other variables are defined as follows.

$$
\begin{aligned}
\boldsymbol{S} & =\boldsymbol{\Phi}^{T} \boldsymbol{Q} \boldsymbol{\Phi}+\boldsymbol{R} \\
\boldsymbol{X}_{1} & =\boldsymbol{\Phi}^{T} \boldsymbol{Q} \boldsymbol{\Psi} \\
\boldsymbol{X}_{2} & =\boldsymbol{\Phi}^{T} \boldsymbol{Q}+\boldsymbol{R} \boldsymbol{H} \\
\overline{\boldsymbol{U}} & =\left[\begin{array}{lll}
\overline{\boldsymbol{u}}^{T} & \ldots & \overline{\boldsymbol{u}}^{T}
\end{array}\right]^{T} \\
\underline{\boldsymbol{U}} & =\left[\begin{array}{lll}
\underline{\boldsymbol{u}}^{T} & \cdots & \underline{\boldsymbol{u}}^{T}
\end{array}\right]^{T} \\
\boldsymbol{\Delta}_{u} & =\left[\begin{array}{lll}
\boldsymbol{\delta}_{u}^{T} & \cdots & \boldsymbol{\delta}_{u}^{T}
\end{array}\right]^{T}
\end{aligned}
$$




$$
\begin{aligned}
& \boldsymbol{E}=\left[\begin{array}{ccccc}
\boldsymbol{I} & \mathbf{0} & \cdots & \mathbf{0} & \mathbf{0} \\
-\boldsymbol{I} & \boldsymbol{I} & \cdots & \mathbf{0} & \mathbf{0} \\
\vdots & \vdots & \cdots & \vdots & \vdots \\
\mathbf{0} & \mathbf{0} & \cdots & -\boldsymbol{I} & \boldsymbol{I}
\end{array}\right] \\
& \boldsymbol{F}=\left[\begin{array}{llll}
\boldsymbol{I} & \mathbf{0} & \cdots & \mathbf{0}
\end{array}\right]^{T}
\end{aligned}
$$

Note, in the above formulation, output constraints are neglected to simplify the algorithm and to fully use the plant capability. The QP problem (5) is efficiently solvable by off-the-shelf software. The only tunable parameters in the above formulation are $Q, \boldsymbol{R}, P, M$ and the sampling time. Thus, the control strategy can be easily implemented and tuned to satisfy required performance.

In vector $\boldsymbol{U}$, only the first $n_{u}$ rows, corresponds to $\boldsymbol{u}(k)$ are applied to the plant. The whole procedure is repeated at the next sampling instance.

For the unconstrained case, the optimal solution, corresponding to a state feedback control law, can be obtained analytically:

$$
\boldsymbol{U}=-\boldsymbol{K}_{1} \boldsymbol{x}(k)+\boldsymbol{K}_{2}\left(\boldsymbol{y}_{r}-\boldsymbol{d}_{k}\right)
$$

where $\boldsymbol{K}_{1}=\boldsymbol{S}^{-1} \boldsymbol{X}_{1}$ and $\boldsymbol{K}_{2}=\boldsymbol{S}^{-1} \boldsymbol{X}_{2}$. Let $\boldsymbol{K}$ be the first $n_{u}$ rows of $\boldsymbol{K}_{1}$, then the nominal stability (perfect model without input saturation) of the closed-loop can be checked by calculating the eigenvalue of the matrix, $\boldsymbol{A}-\boldsymbol{B K}$.

\section{Control design}

\subsection{Predictive control design}

The first task of the control design to implement the above algorithm is to determine an internal model of equation (1). Three operating conditions are specified 
in the gasifier benchmark problem to represent $0 \%, 50 \%$ and $100 \%$ load conditions. All load conditions are subject to disturbance tests. Among these tests, it is observed that those under $0 \%$ load condition are most difficult to pass. That is understandable because a plant at a lower load condition (small throughput) normally exhibits larger time lagging and a larger gain. Therefore, a control system tuned under a higher load condition will tend to be unstable (or stability margin reduced) at a lower load condition. On the other hand, a controller designed for a lower load condition is more likely to work well without re-tuning at higher load conditions although performance might deteriorate when compared with a re-tuned controllers at higher load conditions. Since the performance requirements at 50\% and $100 \%$ load conditions are relatively easier to achieve, it is decided to use the $0 \%$ load point as the nominal point to get the linearized state space model.

A linearized state space model is obtained from the nonlinear simulation model at $0 \%$ load condition. This linear model is then reduced to 16 states via pole-zero cancellation (using Control System Toolbox functions, ssbal and minreal). The 16-state model is then discretized with the sampling time selected as follows.

Normally, the sampling time should be less than one tenth of $2 \pi / \omega_{b}$, where $\omega_{b}$ is the required bandwidth of the closed-loop. The benchmark requires to reject a sine disturbance with a period of 25 seconds $(0.04 \mathrm{~Hz})$. Therefore, the sampling time should be less than 2.5 seconds. On the other hand, the sampling time should not be too large so that in step disturbance tests, the output variables will not deviate from setpoints more than the specified limits before the controller can start to response. Several open-loop tests for a step disturbance of PSINK at three load conditions are performed. The outputs response results are shown in Figure 1. The results show that, the worst response case is the $0 \%$ condition, where, without control, the pressure output can only stay within specified range for a period of 1.2 seconds. Hence, the sampling time is selected to be 1 second. This satisfies the 
requirements for both disturbance tests.

The above algorithm is implemented in MATLAB as a SIMULINK s-function to replace the control block in the nonlinear simulation model provided in the benchmark suit. The QP problem is solved by calling quadprog of the Optimization Toolbox at each sampling time. This is the major computation burden in the above algorithm and is solely determined by the control horizon, $M$. The prediction horizon, $P$ has little effect on computation time, thus can be selected relatively large to benefit stability.

To tune $M$ and $P$, initially let $P=M$. By varying $M$ from $1 \mathrm{~s}$ to $12 \mathrm{~s}$, a stable performance is obtained which satisfies all control specifications for $7 \mathrm{~s} \leq M \leq 10$ s. When $M \geq 10 \mathrm{~s}$, the improvement on the system performance is negligible but computation time increases significantly. Therefor $M=9 \mathrm{~s}$ is selected, which gives a good performance in all tests. To choose a suitable prediction horizon $P$, a reasonable range from the minimum value $(P=M=9 \mathrm{~s})$ to $P=25 \mathrm{~s}$ has been tested. A stable response without any constraint violation is found within the range $15 \mathrm{~s} \leq P \leq 20 \mathrm{~s}$. No performance improvement can be observed when $P \geq 20 \mathrm{~s}$. Therefore $P=20 \mathrm{~s}$ (the maximum value of the range) is chosen to ensure both the system stability and satisfactory control performance achieved within a reasonable computation time.

The weighting matrix, $\boldsymbol{Q}=\operatorname{diag}\left(\boldsymbol{Q}_{0}, \cdots, \boldsymbol{Q}_{0}\right)$, where $\boldsymbol{Q}_{0}$ is diagonal and initially set to be the inverse of the output error bounds. After online tuning, the final values are:

$$
\boldsymbol{Q}_{0}=\left[\begin{array}{cccc}
0.15 & 0 & 0 & 0 \\
0 & 100 & 0 & 0 \\
0 & 0 & 2.1 & 0 \\
0 & 0 & 0 & 2 \times 10^{6}
\end{array}\right]
$$


Also, the input weighting matrix $\boldsymbol{R}=\operatorname{diag}\left(\boldsymbol{R}_{0}, \cdots, \boldsymbol{R}_{0}\right)$, where $\boldsymbol{R}_{0}$ is diagonal and set to the following value after online tuning;

$$
\boldsymbol{R}_{0}=\left[\begin{array}{cccc}
10^{5} & 0 & 0 & 0 \\
0 & 5 \times 10^{3} & 0 & 0 \\
0 & 0 & 5 \times 10^{3} & 0 \\
0 & 0 & 0 & 10^{4}
\end{array}\right]
$$

Using the above configuration, nominal stability is achieved at all three load conditions, i.e. the magnitudes of all eigenvalues of $\boldsymbol{A}_{i}-\boldsymbol{B}_{i} \boldsymbol{K}$ are less than 1. Where, $\boldsymbol{A}_{i}$ and $\boldsymbol{B}_{i}$ are the discrete states and control matrices at different load conditions.

One of the advantages of MPC is that future setpoint change information is incorporable into the QP optimization problem to improve setpoint tracking performance. This is implemented in the gasifier controller.

\section{Simulation results}

\subsection{Disturbance tests}

The following two disturbance tests are performed for three load conditions for 300 seconds:

1. a step change of -0.2 bar in sink pressure (PSINK) at $30 \mathrm{~s}$;

2. a $0.04 \mathrm{~Hz}$ sinusoidal variation of amplitude 0.2 bar in PSINK beginning at $30 \mathrm{~s}$.

The maximum and minimum values as well as the peak rate change of the input variables of the two tests under different load conditions are shown in Table 1.

The maximum absolute error between output variables and the corresponding setpoints and the integral of absolute error (IAE) of these variables are given in 
Table 2. Plots of output and input responses to disturbances at $0 \%$ load conditions are shown in Figures 2 to 5. For sinusoidal disturbance test, the results with extra simulation time $(1500 \mathrm{~s})$ are provided to confirm the satisfactory of performance specifications.

The output response plots together with Tables 1 and 2 show that the predictive controller based on a linear model identified at $0 \%$ load is able to keep the output variables within the limits specified for both tests. Particularly, the performance at $0 \%$ load is significantly improved from the one achieved by the PID configuration provided in the benchmark. However, this improvement is traded with the price of performance deterioration at other load conditions although the performance specifications are still satisfied at those load conditions.

\subsection{Load change test}

In this test, the load is required to increase from $50 \%$ to $100 \%$ within a time period $100 \mathrm{~s}$ to $700 \mathrm{~s}$. Output, Input and load response data are collected during simulation and compared with the demand in Figures 6 and 7. Significant improvement (comparing with the results using the PID controller provided in the benchmark) in the setpoint tracking performance can be observed in the results. This improvement is due to the advantage of predictive control to wisely use future setpoint information to obtain the best moves in the online optimization.

\subsection{Coal quality change test}

The benchmark problem includes a test of coal quality changes by $\pm 18 \%$. Physically, a positive coal quality change means an increase of energy per unit coal feed. To maintain the same level of load, it is expectable that coal feed and char outlet (ash) will decrease at steady state due to energy balance. Similarly, a negative coal quality change will increase coal feed and char outlet at steady state. Therefore, 
the feasible range of a coal quality change is restricted by the input constraints. If a coal quality change is beyond this feasible range, at steady-state some input saturations are inevitable and the control problem becomes infeasible, i.e. there is no controller which can achieve the performance specification.

Since an analytical model is no available, the feasible coal quality range is determined via simulation described as follows. Set the PID simulation model provided in the benchmark by removing all actuator constraints. Then repeatedly perform simulation until steady state by introducing a different coal quality change but without any other disturbance. By checking the steady-state input values against their constraints the following feasible coal quality ranges for different load conditions are identified.

100\% load $-6.6 \% \leq$ coal quality $\leq+7.1 \%$

$\mathbf{5 0 \%}$ load $-14.1 \% \leq$ coal quality $\leq+11.3 \%$

0\% load $-16 \% \leq$ coal quality $\leq+18 \%$

At the upper bounds of feasible coal quality ranges, WCHR reaches its lower bound at steady stated, whilst at the lower bounds of the feasible ranges WCHR saturates at its upper bound except at 100\% load condition where WCOL, instead of WCHR, becomes saturated at its upper bound. The above results show that a performance deterioration is inevitable when a coal quality change is out of the above feasible range. This deterioration is independent of control design because of the inherent limitation imposed by the physical nature of the system.

Such performance deterioration is also observed for the MPC controller. It has been identified that the predictive controller can cope with coal quality change of $\pm 18 \%$ for all standard disturbance tests for up to 600 seconds. For simulation time longer than 600 seconds, output specifications are violated in sinusoidal tests under $0 \%$ load with a coal quality change of $-18 \%$ and under $100 \%$ load with a coal 
quality change of $+18 \%$. Steady state test for a longer time (for example 15000 seconds) shows that the system cannot cope with a coal quality change either of $-18 \%$ under $0 \%$ and $50 \%$ load conditions, or $+18 \%$ under $50 \%$ and $100 \%$ load conditions.

In the presence of sinusoidal disturbance test at $100 \%$ load, for positive coal quality change, WCHR tends to zero at $t \geq 200 \mathrm{~s}$ as shown in figure 9. When this happens for sufficiently long time, temperature starts to go up as more carbon has to be burned to balance high coal quality inlet (figure 8). Similarly, for sufficiently negative coal quality change, WCHR, and WCOL will be saturated at their upper bounds. The gasification process in this case is under combusted and outlet gas temperature will unavoidably drop. A working solution is to change output setpoint when an input saturation is detected so that the gasifier can maintain the same load demand but without WCHR or WCOL saturations. This however is not within the specifications of the benchmark problem and hence will not be discussed further.

\section{Conclusions}

A simple predictive controller has been developed to control the ALSTOM gasifier process. By using a linear state space model identified at $0 \%$ load condition as the internal model, the controller is able to achieve all required performance specifications within the input and output constraints. The performance deterioration at $50 \%$ and $100 \%$ load conditions due to using a model linearized at $0 \%$ load point although small, indicates that using a nonlinear predictive model might be necessary to improve performance at all load conditions. A prototype controller in Simulink has been developed within a week, whilst tuning and simulation take another week to complete. The success of this case study reveals that nominal model selection could be an important issue when using a linear model-based controller to control 
nonlinear systems. Some theoretical work may have to be done in order to guide how to select an appropriate linear nominal model for nonlinear system control in general.

\section{References}

[1] R. Dixon, A. Pike, and M. Donne, "The ASTOM benchmark challenge on gasifier control," Proc. Instn Mech. Engrs. Part I, Journal of Systems and Control Engineering, vol. 214, pp. 389-394, 2000.

[2] R. Dixon, "Advanced gasifier control," Computing and Control Engineering Journal, IEE, vol. 10, no. 3, pp. 93-96, 1999.

[3] M. Rice, J. Rossiter, and J. Schurmans, "An advanced predictive control approach to the ASTOM gasifier problem," Proc. Instn Mech. Engrs. Part I, Journal of Systems and Control Engineering, vol. 214, pp. 405-413, 2000.

[4] C. Chin and N. Munro, "Control of the ALSTOM gasifier benchmark problem using $\mathrm{H}_{2}$ methodology," Journal of Process Control, vol. 13, pp. 759-768, 2003.

[5] R. Dixon and A. W. Pike, "ALSTOM benchmark challenge on gasifier control", IEE Proc. Control Theory and Applications, under review for the special issue/section on gasifier control. 


\section{List of Figures}

1 Open-loop output response to a step disturbance at 30s at $0 \%$ (solid), $50 \%$ (dashed) and 100\% (dash-dotted) load conditions. . . . . . 16

2 Output response to step disturbance at $0 \%$ load. . . . . . . . . . 17

3 Input response to step disturbance at $0 \%$ load. . . . . . . . 18

4 Output response to sinusoidal disturbance at $0 \%$ load. . . . . . . . 19

5 Input response to sinusoidal disturbance at $0 \%$ load. . . . . . . . . 20

6 Output responses to a load setpoint change . . . . . . . . . 21

7 Input responses to a load setpoint change . . . . . . . . . . 22

8 Output response to a coal quality change of $18 \%$ in $100 \%$ load

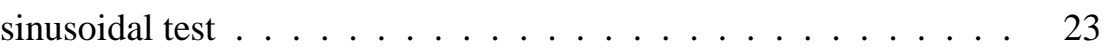

9 Input response to a coal quality change of $18 \%$ in $100 \%$ load sinusoidal test . . . . . . . . . . . . . . . . . 24 

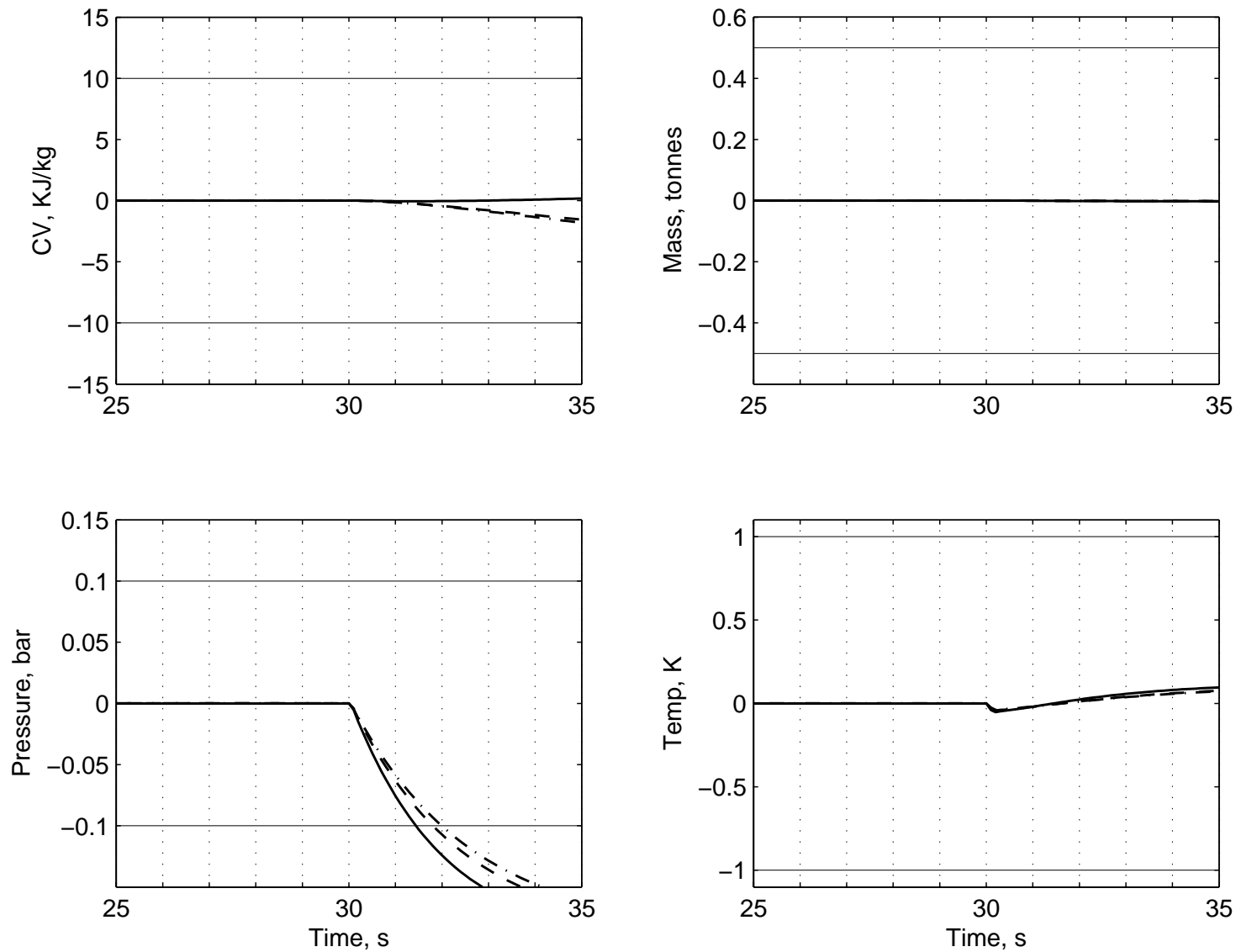

Figure 1: Open-loop output response to a step disturbance at 30s at $0 \%$ (solid), $50 \%$ (dashed) and 100\% (dash-dotted) load conditions. 

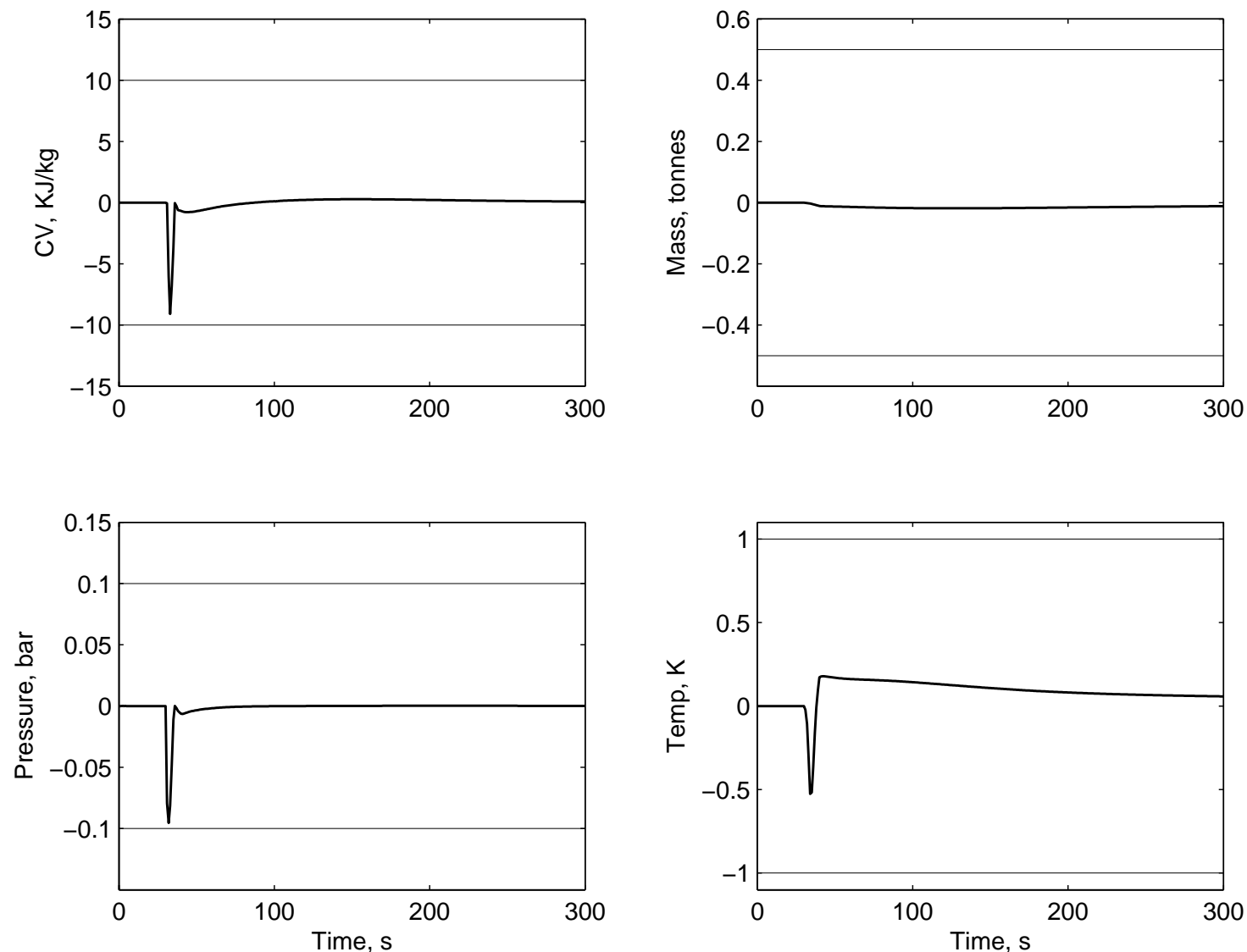

Figure 2: Output response to step disturbance at $0 \%$ load. 

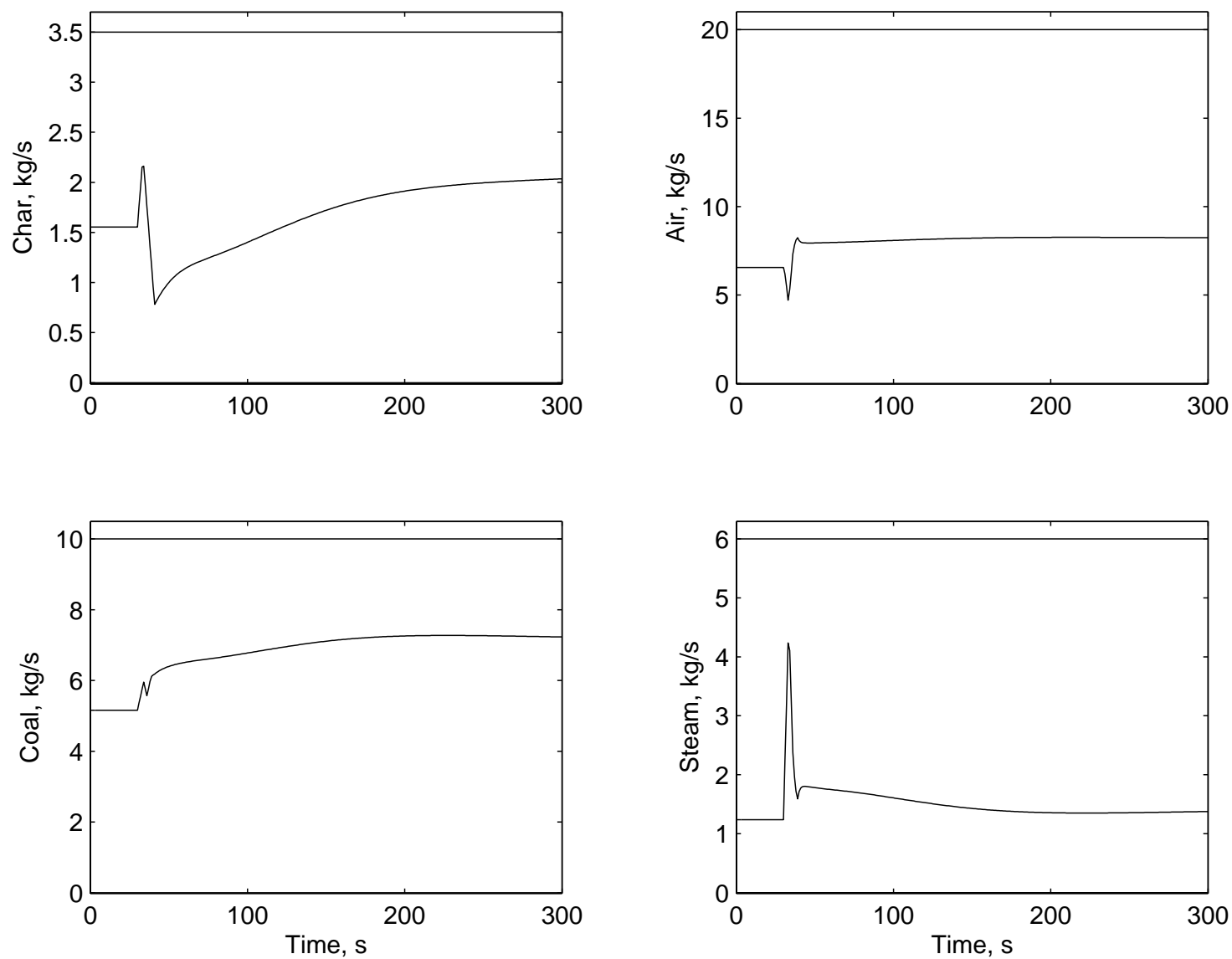

Figure 3: Input response to step disturbance at $0 \%$ load. 

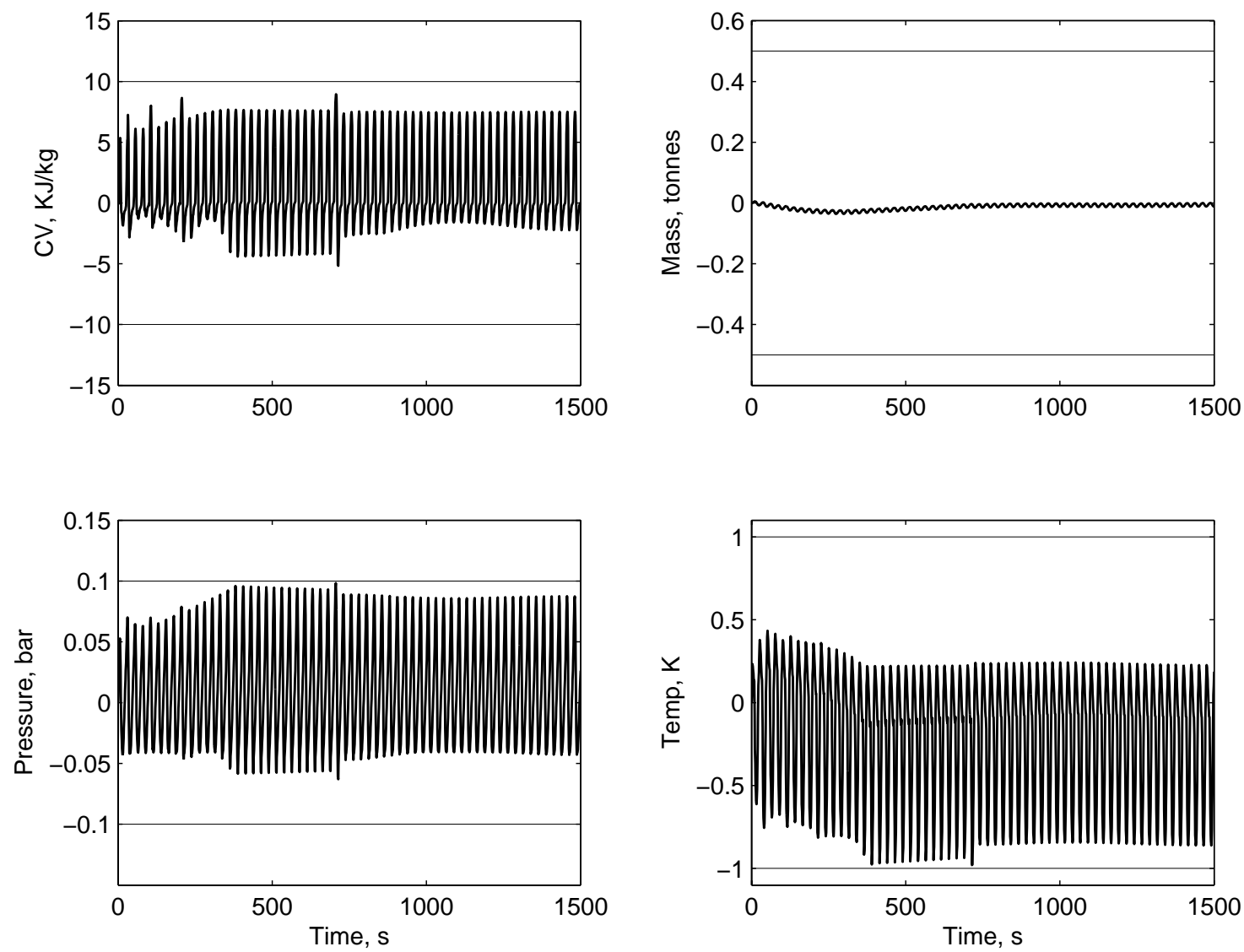

Figure 4: Output response to sinusoidal disturbance at $0 \%$ load. 

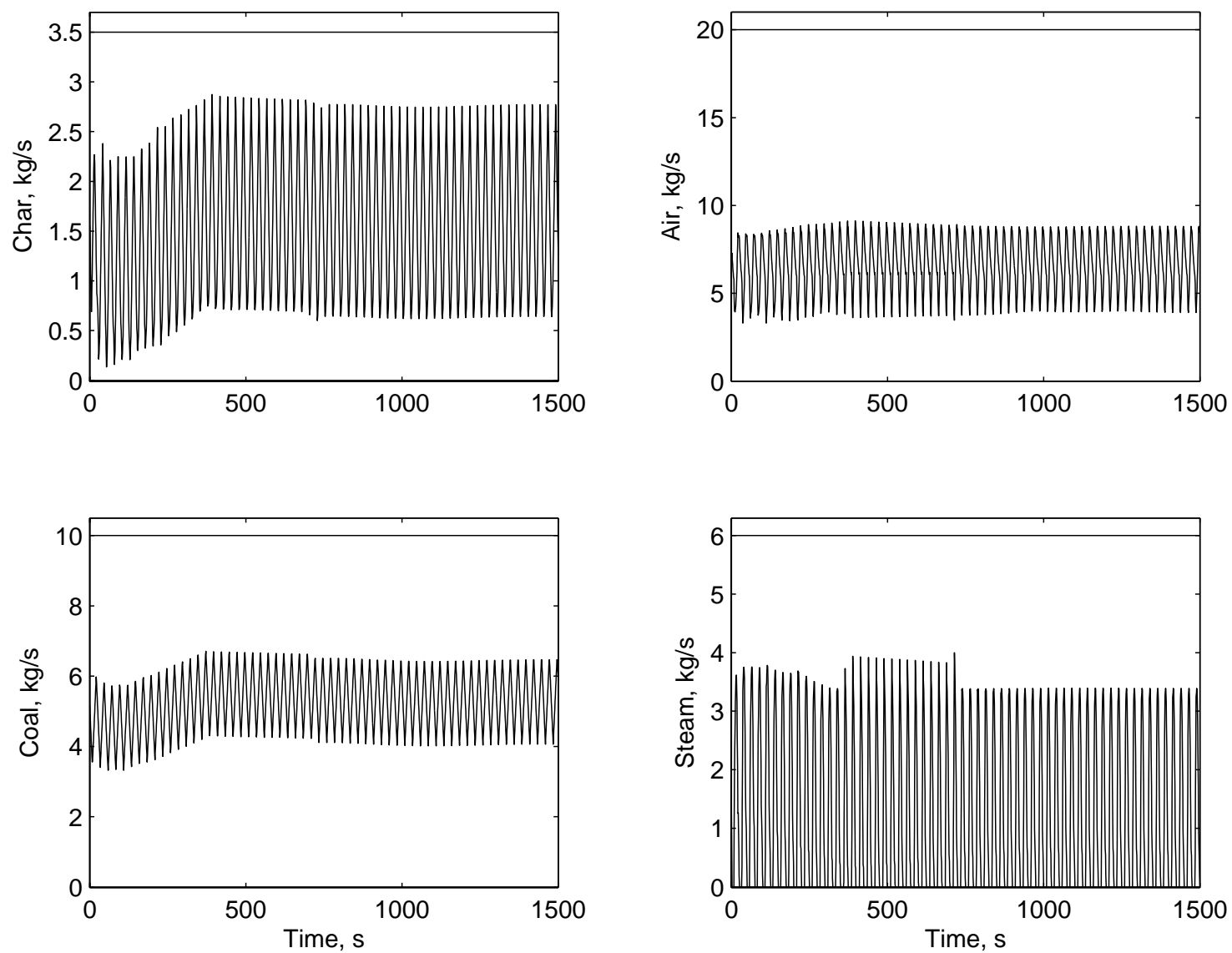

Figure 5: Input response to sinusoidal disturbance at $0 \%$ load. 

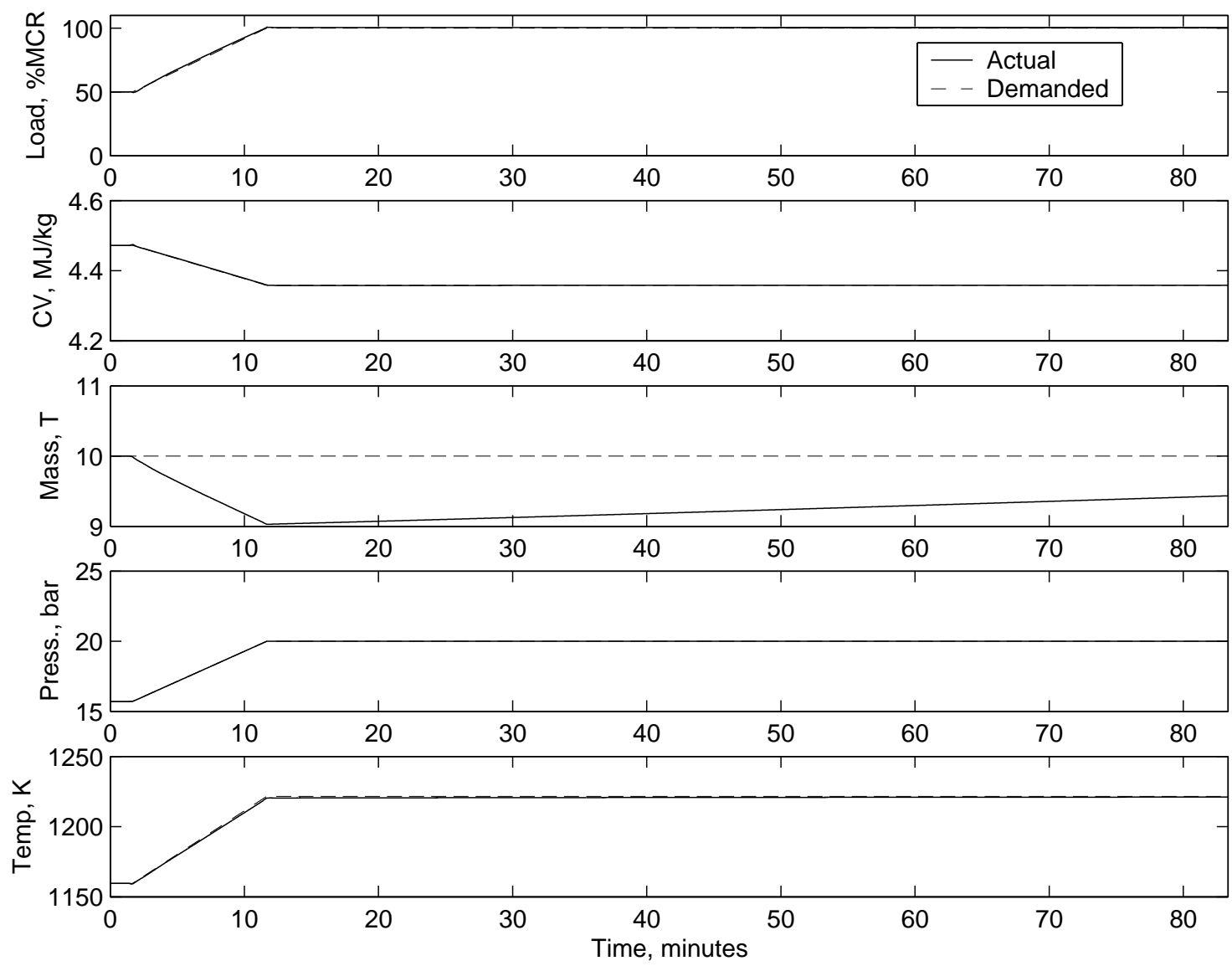

Figure 6: Output responses to a load setpoint change 

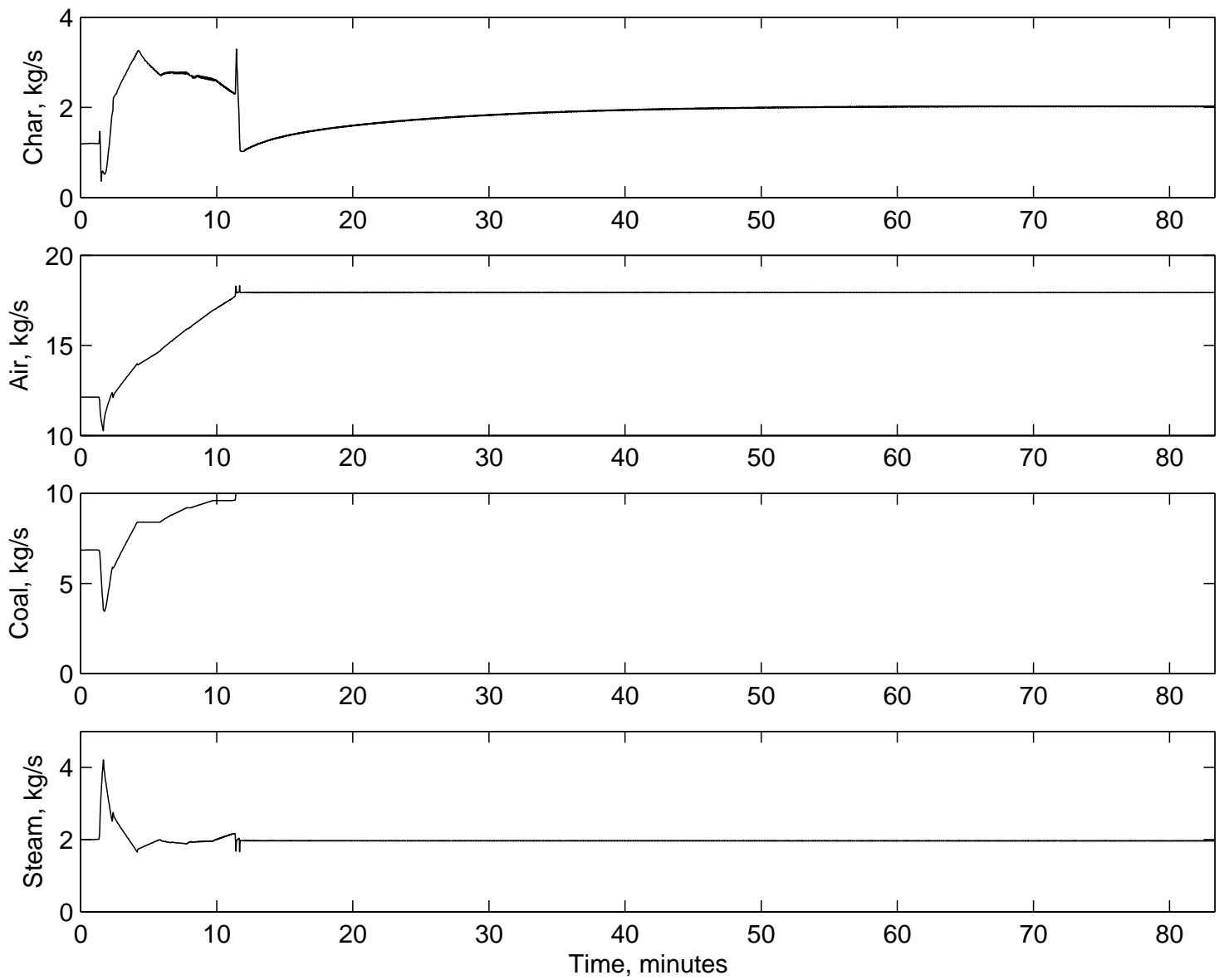

Figure 7: Input responses to a load setpoint change 

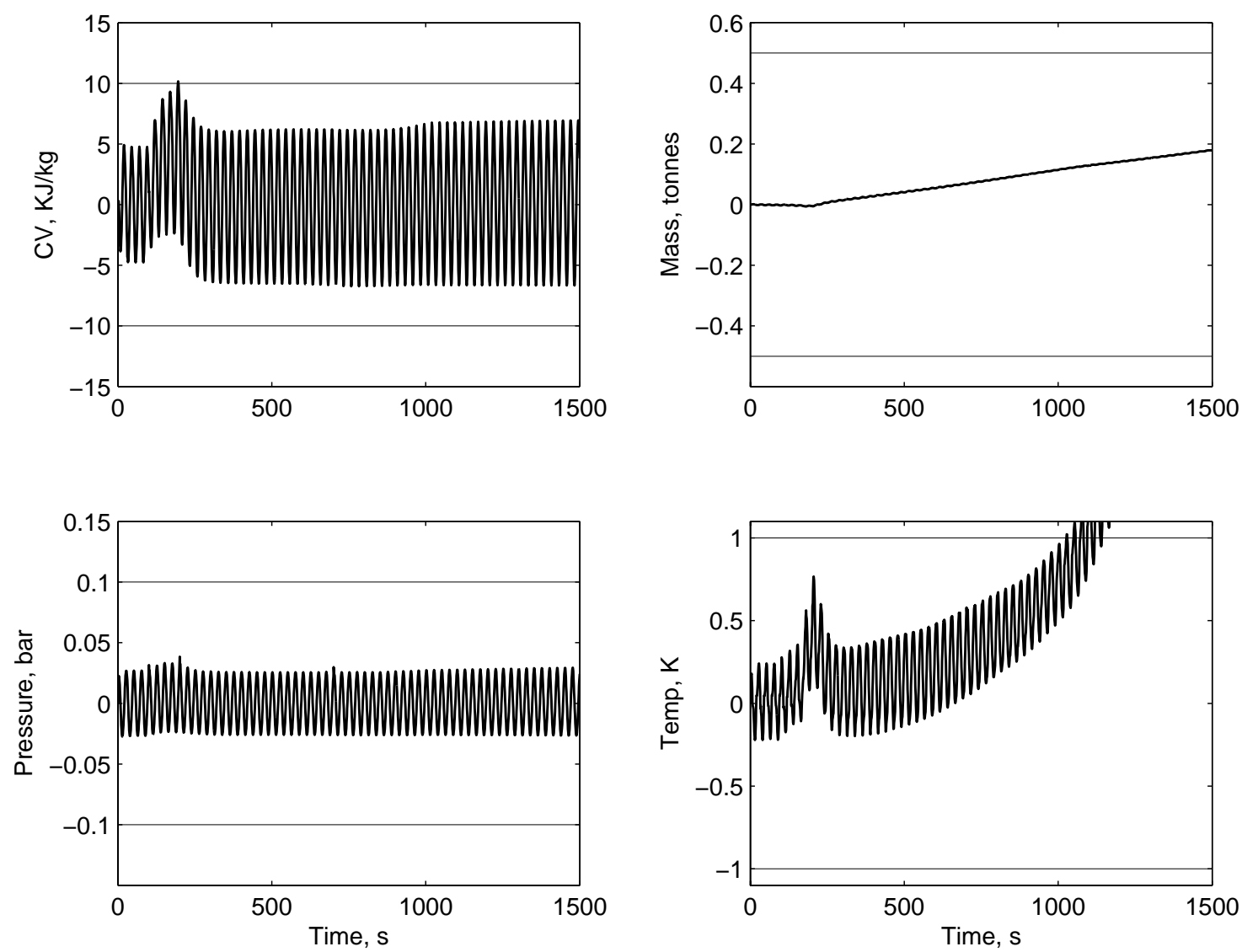

Figure 8: Output response to a coal quality change of $18 \%$ in $100 \%$ load sinusoidal test 

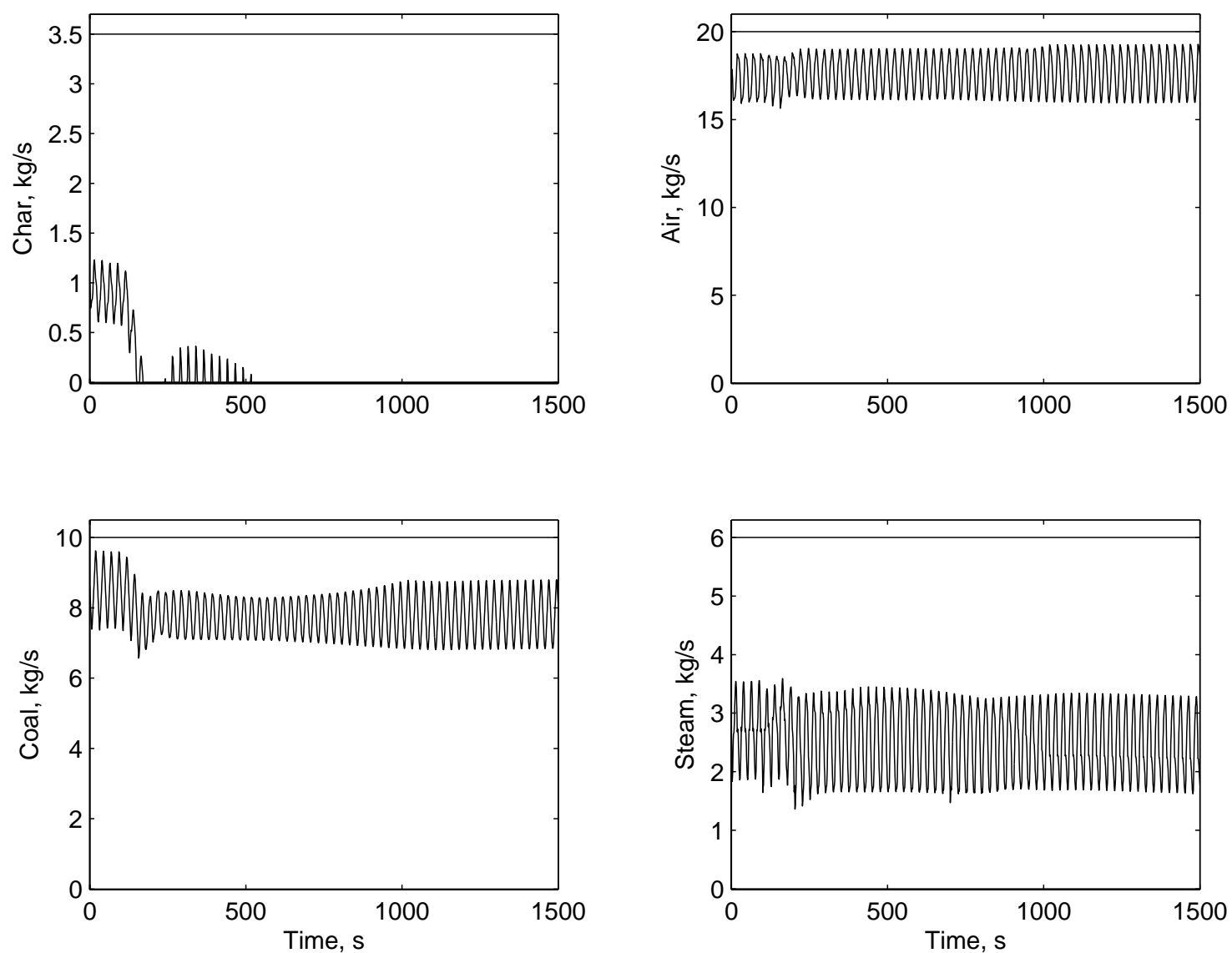

Figure 9: Input response to a coal quality change of $18 \%$ in $100 \%$ load sinusoidal test 
Table 1: Input results

\begin{tabular}{cccc}
\hline Step, 100\% load & Maximum & Minimum & Peak rate \\
\hline WCHR & 1.585 & 0.435 & 0.2 \\
WAIR & 19.071 & 16.136 & 1 \\
WCOL & 10.000 & 8.619 & 0.2 \\
WSTM & 5.109 & 2.531 & 1 \\
\hline Step, 50\% load & Maximum & Minimum & Peak rate \\
\hline WCHR & 1.796 & 0.583 & 0.2 \\
WAIR & 13.934 & 10.360 & 1 \\
WCOL & 8.653 & 6.845 & 0.2 \\
WSTM & 4.992 & 1.897 & 1 \\
\hline Step, 0\% load & Maximum & Minimum & Peak rate \\
\hline WCHR & 2.162 & 0.781 & 0.2 \\
WAIR & 8.264 & 4.714 & 1 \\
WCOL & 7.274 & 5.157 & 0.2 \\
WSTM & 4.237 & 1.237 & 1 \\
\hline Sine, 100\% load & Maximum & Minimum & Peak rate \\
\hline WCHR & 1.300 & 0.581 & 0.125 \\
WAIR & 18.806 & 15.920 & 0.517 \\
WCOL & 9.730 & 7.371 & 0.2 \\
WSTM & 3.557 & 1.611 & 0.603 \\
\hline Sine, 50\% load & Maximum & Minimum & Peak rate \\
\hline WCHR & 1.597 & 0.672 & 0.119 \\
WAIR & 13.704 & 10.393 & 0.606 \\
WCOL & 8.021 & 5.445 & 0.2 \\
WSTM & 3.297 & 0.679 & 0.684 \\
\hline Sine, 0\% load & Maximum & Minimum & Peak rate \\
\hline WCHR & 2.669 & 0.134 & 0.2 \\
WAIR & 8.964 & 3.303 & 1 \\
WCOL & 6.406 & 3.321 & 0.2 \\
WSTM & 3.782 & 0 & 1 \\
\hline & & &
\end{tabular}


Table 2: Output results

\begin{tabular}{ccc}
\hline Step, 100\% load & Maximum absolute error & IAE \\
\hline CVGAS & 7.685 & 90.975 \\
MASS & 12.915 & - \\
PGAS & 0.0674 & 0.251 \\
TGAS & 0.529 & - \\
\hline Step, 50\% load & Maximum absolute error & IAE \\
\hline CVGAS & 7.370 & 75.022 \\
MASS & 8.046 & - \\
PGAS & 0.076 & 0.308 \\
TGAS & 0.610 & - \\
\hline Step, 0\% load & Maximum absolute error & IAE \\
\hline CVGAS & 9.084 & 86.041 \\
MASS & 18.504 & - \\
PGAS & 0.095 & 0.458 \\
TGAS & 0.525 & - \\
\hline Sine, 100\% load & Maximum absolute error & IAE \\
\hline CVGAS & 4.911 & 868.240 \\
MASS & 2.772 & - \\
PGAS & 0.0313 & 5.178 \\
TGAS & 0.273 & - \\
\hline Sine, 50\% load & Maximum absolute error & IAE \\
\hline CVGAS & 4.274 & 713.473 \\
MASS & 5.290 & - \\
PGAS & 0.033 & 5.773 \\
TGAS & 0.323 & - \\
\hline Sine, 0\% load & Maximum absolute error & IAE \\
\hline CVGAS & 8.645 & 657.218 \\
MASS & 35.783 & - \\
PGAS & 0.083 & 10.110 \\
TGAS & 0.814 & - \\
\hline
\end{tabular}

